\title{
Does duration of DGF affect graft outcome after DCD donor kidney transplantation?
}

\author{
Awad Shamali, Theodoros Kassimatis, Hannah Burton, Nicos Kessaris, Chris Callaghan \\ Department of Nephrology and Transplantation, Guy's Hospital, London, UK
}

Introduction: In the UK, outcomes of kidney transplants from donation after circulatory death (DCD) donors are equivalent to those from donation after brain death (DBD) donors. Delayed graft function (DGF) is approximately twice as common after DCD donor kidney transplantation, however. The impact of duration of DGF is poorly defined. A retrospective analysis of our DCD donor kidney transplant programme was performed to determine if duration of DGF affected graft outcomes.

Method: Single kidney-only grafts from controlled DCD donors transplanted into adult recipients between 2011-2016 were analysed. DGF was defined as the need for dialysis (any cause) within the first week of transplantation. Duration of DGF was defined as the number of days from transplantation to last dialysis session. Outcome measures included 6, 12, 24 and 36 month eGFR.' Recipients with DGF were divided into three groups based on DGF duration (group I - DGF <7 days; group II - DGF 7-14 days; group III - DGF $>14$ days).

Results: 236 out of 245 DCD recipients were included; 93 $(39.4 \%)$ had immediate graft function. DGF occurred in 143 (60.6\%) recipients: group I - 75 (31.8\%); group II - 45 (19.1\%); group III - 23 (9.7\%). Median (range) donor age was 54 (6-79) years, median recipient age was 53 (18-79) years, median cold ischaemic time (CIT) was 790 (340-1520) minutes (Table 1). Overall, DGF was associated with a higher rate of graft failure than those recipients that had no DGF $(11.2 \%$ versus $0 \%, p=0.001)$. Graft function was worse in the DGF group during the first 6 and 12 months of follow up, $(p=0.005$ and 0.049 respectively). However, no difference in graft function was found during 24 and 36 months between the DGF and non-DGF groups $(p=0.192$, and 0.615 respectively).

Comparing groups I, II, and III, the duration of DGF was not associated with an increasing rate of graft failure, $p=0.563$ (see graph below). Meanwhile the duration of DGF was found to be associated with worse graft function at 6 , 12 , and 36 months follow-up $(p=0.002,0.008$, and 0.026 respectively - Table 2).

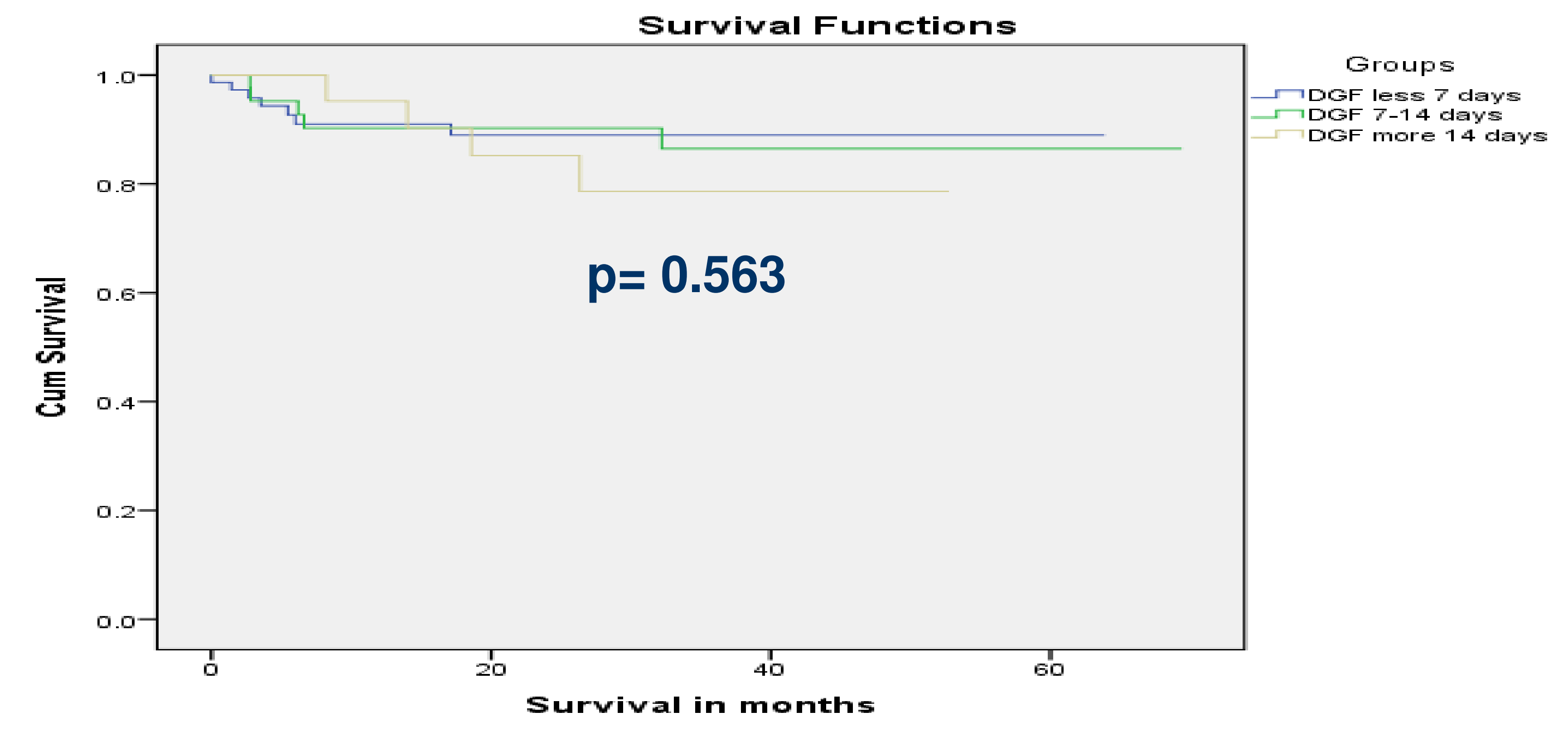

Table 1: Group characteristics

\begin{tabular}{|c|c|c|c|c|}
\hline & Group I & Group II & Group III & $\mathbf{P}$ \\
\hline Number & 75 & 45 & 23 & \\
\hline Recipient sex M/F & $58 / 17$ & $31 / 14$ & $15 / 8$ & 0.409 \\
\hline $\begin{array}{l}\text { Recipient age at } \\
\text { transplant }\end{array}$ & $58(47-63)$ & $57(48-62)$ & $51(43-61)$ & 0.377 \\
\hline $\begin{array}{l}\text { Recipient black } \\
\text { ethnicity }\end{array}$ & $37(55.2)$ & $16(23.9)$ & $14(20.9)$ & 0.116 \\
\hline Donor sex $M / F$ & $45 / 30$ & $34 / 11$ & $12 / 11$ & 0.106 \\
\hline Donor hypertension & $26(34.7)$ & $11(24.4)$ & $7(30.4)$ & 0.748 \\
\hline Donor diabetes mellitus & $4(5.3)$ & $3(6.7)$ & $0(0)$ & 0.841 \\
\hline Donor adrenaline use & $2(2.7)$ & $2(4.4)$ & $1(4.3)$ & 0.875 \\
\hline Donor age & $54(49-65)$ & $56(50-65)$ & $56(48-65)$ & 0.908 \\
\hline Donor BMI & $25(23-28)$ & $26(23-30)$ & $28(23-33)$ & 0.453 \\
\hline $\begin{array}{l}\text { Donor terminal } \\
\text { creatinine }\end{array}$ & $\begin{array}{c}74(58- \\
104)\end{array}$ & $76(49-96)$ & $\begin{array}{c}53(45- \\
104)\end{array}$ & 0.272 \\
\hline Donor, UK-KDRI & $\begin{array}{c}1.36 \\
(0.99-1.58)\end{array}$ & $\begin{array}{c}1.29 \\
(1.02-1.57)\end{array}$ & $\begin{array}{c}1.21 \\
(1.03-1.88)\end{array}$ & 0.924 \\
\hline Donor, US-KDRI & $\begin{array}{c}1.69 \\
(1.25-2.08)\end{array}$ & $\begin{array}{c}1.45 \\
(1.22-1.88)\end{array}$ & $\begin{array}{c}1.40 \\
(1.13-2.05)\end{array}$ & 0.374 \\
\hline Cold ischaemic time & $\begin{array}{c}810 \\
(612-990)\end{array}$ & $\begin{array}{c}780 \\
(635-1003)\end{array}$ & $\begin{array}{c}945 \\
(685-1105)\end{array}$ & 0.413 \\
\hline
\end{tabular}

Table 2: Graft outcomes

\begin{tabular}{l|c|c|c|c} 
& Group I & Group II & Group III & P \\
\hline Number & 75 & 45 & 23 & \\
Graft failure & $7(9.3)$ & $5(11.1)$ & $4(17.4)$ & 0.563 \\
$\begin{array}{l}\text { Biopsy proven acute } \\
\text { rejection }\end{array}$ & $11(14.7)$ & $12(26.7)$ & $10(43.5)$ & 0.013 \\
\hline eGFR 6 months & $47(36-64)$ & $39(31-57)$ & $26(19-45)$ & 0.002 \\
\hline eGFR 12 months & $50(41-64)$ & $42(35-57)$ & $32(21-58)$ & 0.008 \\
\hline eGFR 24 months & $54(43-76)$ & $49(34-63)$ & $35(29-63)$ & 0.053 \\
\hline eGFR 36 months & $58(47-73)$ & $47(31-58)$ & $33(23-61)$ & 0.026
\end{tabular}

Conclusion: DGF was frequent in our DCD donor kidney transplant programme, and often lasted more than 7 days. Prolonged DGF was associated with progressively worse short and medium-term graft function. This may be due to higher rates of BPAR, though it is difficult to distinguish cause from effect. Duration of DGF did not appear to adversely impact on death-censored graft survival, though this is likely due to lack of long-term follow-up. Interventions to reduce DGF rates are needed.

\section{Guy's and St Thomas' WHS NHS Foundation Trust}

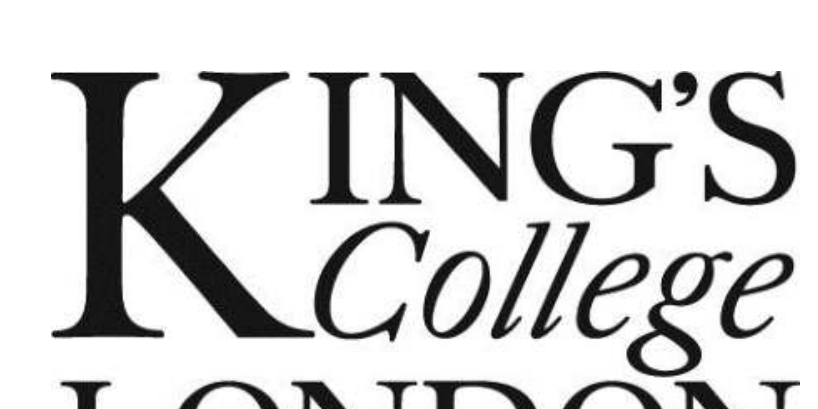
LONDON www.guysandstthomas.nhs.uk

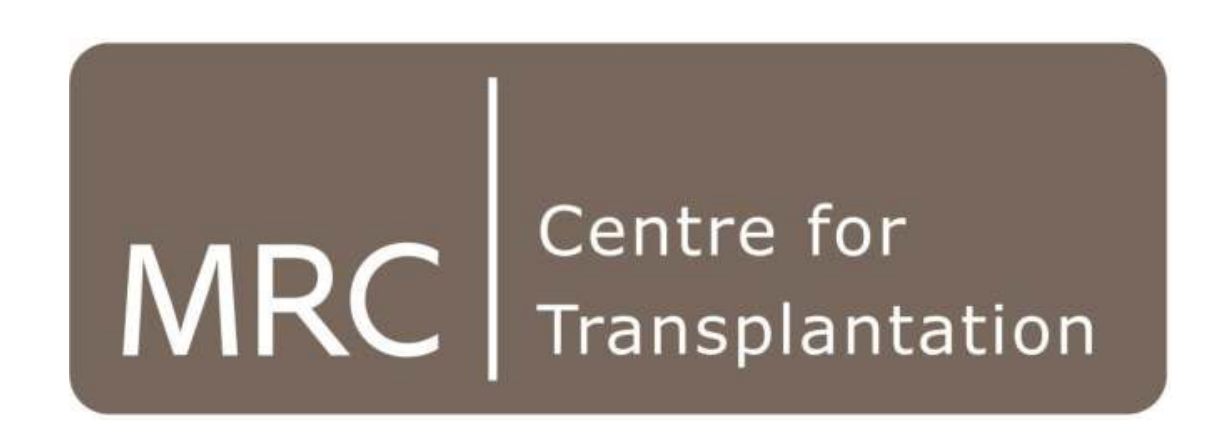

www.kcl.ac.uk

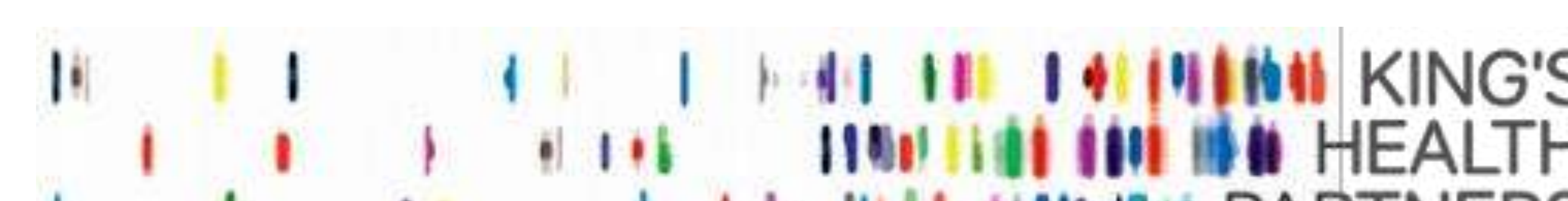 I I i in il ililli ini PARTNERS www.kingshealthpartners.org

